\title{
Biologics may have a beneficial effect in asthma patients with COVID-19
}

\begin{abstract}
Reply to P.A.B. Wark and co-workers:
Copyright (CThe authors 2021

This version is distributed under the terms of the Creative Commons Attribution NonCommercial Licence 4.0. For commercial reproduction rights and permissions contact permissions@ersnet.org

Received: 13 April 2021 Accepted: 24 June 2021

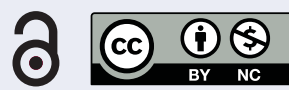

Shareable abstract (@ERSpublications)

Biologics may have a beneficial effect in asthma patients with COVID-19 https://bit.ly/3hkor8X

Cite this article as: Izquierdo JL, Soriano JB. Biologics may have a beneficial effect in asthma patients with COVID-19. Eur Respir J 2021; 58: 2101076 [DOI: 10.1183/13993003.01076-2021].

José L. Izquierdo ${ }^{1,2}$ and Joan B. Soriano $\circledast^{3,4,5}$

${ }^{1}$ Universidad de Alcalá, Madrid, Spain. ${ }^{2}$ Hospital Universitario de Guadalajara, Guadalajara, Spain. ${ }^{3}$ Hospital Universitario de La Princesa, Madrid, Spain. ${ }^{4}$ Universidad Autónoma de Madrid, Madrid, Spain. ${ }^{5}$ Centro de Investigación en Red de Enfermedades Respiratorias (CIBERES), Instituto de Salud Carlos III (ISCIII), Madrid, Spain.
\end{abstract}

Corresponding author: Joan B. Soriano (jbsoriano2@gmail.com)

Conflict of interest: J.L. Izquierdo reports personal fees from AstraZeneca, Bayer, Boehringer Ingelheim, Chiesi, GSK, Grifols, Menarini, Novartis, Orion, Pfizer, Sandoz and Teva. J.B. Soriano has nothing to disclose.

\section{References}

1 Izquierdo JL, Almonacid C, González Y, et al. The impact of COVID-19 on patients with asthma. Eur Respir J 2021; 57: 2003142.

2 Pala D, Pistis M. Anti-IL5 drugs in COVID-19 patients: role of eosinophils in SARS-CoV-2-induced immunopathology. Front Pharmacol 2021; 12: 622554. 